\title{
Casas resilientes en Puerto Rico: resistir al desastre redefiniendo la vivienda
}

Resilient housing in Puerto Rico: resisting disaster by redefining housing

\section{Omayra Rivera-Crespo}

Universidad Politécnica de Puerto Rico (Puerto Rico)

Escuela de Arquitectura

\section{Yara Colón Rodríguez}

Universidad Politécnica de Puerto Rico (Puerto Rico)

Escuela de Arquitectura
Rivera-Crespo, 0., \& Colón Rodríguez, Y. (202I). Casas resilientes en Puerto Rico: resistir al desastre redefiniendo la vivienda. Revista de Arquitectura (Bogotá), 23(2), 84-93. https://doi.org/10.14718/ RevArq.2021.2793

\author{
Omayra Rivera-Crespo \\ Doctora en Arquitectura, Escuela de Arquitectura La Salle, Universidad \\ Ramon Llull. Barcelona, España. \\ Maestría en Arquitectura, Arizona State University. Arizona, EE. UU. \\ Arquitecta, Escuela de Arquitectura, Universidad de Puerto Rico. San Juan, \\ Estado Libre Asociado de Puerto Rico. \\ Posgrado en Arquitectura, Arte y Espacio Efímero, Universidad Politécnica \\ de Catalunya. Barcelona, España. \\ Autora del libro Procesos de participación: proyectar, construir y habitar la \\ vivienda contemporánea. \\ Cofundadora del colectivo Taller Creando Sin Encargos. \\ Profesora en Boston, Barcelona y Puerto Rico. Actualmente continúa tra- \\ bajando como docente, gerente y coordinadora de proyectos, y como \\ consultora de diseño participativo. \\ (D) https://orcid.org/0000-0002-6|48-5537 \\ - https://scholar.google.es/citations?user=x-gnurEAAAAJ\&hl=es \\ (10) omayra.rivera.crespo@gmail.com \\ Yara Colón Rodríguez \\ Doctora en Teoría e Historia de la Arquitectura, Universitat Politècnica de \\ Catalunya. Barcelona, España. \\ Maestría en Historia, Arte, Arquitectura y Ciudad, Universitat Politècnica \\ de Catalunya. Barcelona, España. \\ Posgrado en Estética y Teoría del Arte Contemporáneo, Universitat Autó- \\ noma de Barcelona. Barcelona, España. \\ Bachillerato en Arquitectura y Humanidades, Universidad de Puerto Rico. \\ San Juan, Estado Libre Asociado de Puerto Rico. \\ Investigadora, historiadora y profesora de historia, teoría e historiografía \\ de la arquitectura o el diseño. \\ Editora, revista Polimorfo. \\ Cofundadora de la Colectiva para la Investigación de la Historia y Teoría de \\ la Arquitectura y el Diseño en Puerto Rico. \\ (D) https://orcid.org/0000-0002-5972-754| \\ https://scholar.google.com/citations?user=8uJ|r9sAAAA|\&hl=es \\ (10yaramaite@gmail.com
}

\section{Resumen}

El propósito de este artículo de reflexión es indagar sobre el concepto de resiliencia, como potenciador de resistencia en el caso de la vivienda y en un contexto posdesastre, natural y socioeconómico, en Puerto Rico. La resiliencia/resistencia se proponen como dos estadios de un proceso posible, radical y esperanzador. Algunos de los objetivos son: demostrar cómo es una casa resiliente/resistente, qué cualidades la constituyen y cómo esta puede posibilitar formas reales de resistencia física y social. La revisión bibliográfica asiste en la definición del problema y la pertinencia de la investigación, además de aportar algunas consideraciones teóricas sobre el desastre, la resiliencia y la casa, y en proveer referencias con las cuales ampliar la interpretación. La metodología consiste, principalmente, en el análisis de procesos de agenciamiento del espacio y proyectos de vivienda en Puerto Rico que han buscado resistir ante el desastre. Como parte de los resultados, se trazan parámetros para que la casa pueda resistir al desastre en todas sus facetas.

Palabras clave: calidad de vida; asentamiento humano; diseño de vivienda; reconstrucción; adaptación al cambio climático

\begin{abstract}
The purpose of this reflection article is to investigate the concept of resilience as a resistance enhancer in the case of housing in a natural and socioeconomic post-disaster context, in Puerto Rico. Resilience/resistance are proposed as two stages of a possible, radical and hopeful process. The objectives of this paper are: to demonstrate what a resilient/resistant house looks like, what qualities constitute it and how it can enable real forms of physical and social resistance. The bibliographic review supports the definition of the problem and the relevance of the research, in addition to contributing some theoretical considerations on disaster, resilience and housing, and providing references to broaden the interpretation. The methodology consists mainly of the analysis of spatial agency processes and housing projects in Puerto Rico seeking to resist disaster. As part of the results, parameters are outlined so that the house can resist the disaster in all its facets.
\end{abstract}

Keywords: climate change adaptation; human settlements; housing design; quality of life; reconstruction

\section{Introducción}

La siguiente investigación se desarrolla como parte de una iniciativa independiente, y surge del interés en investigar la vivienda posdesastre en Puerto Rico, sus características y los proyectos locales existentes. El artículo tiene como punto de partida la presentación titulada Casas Resilientes en Puerto Rico: repensando la vivienda después del huracán María, presentada en el Ciclo de Conferencias Arquitectonics: Mind, Land and Society, de la Universidad Politécnica de Cataluña, en Barcelona, entre mayo y junio de 2018.

El estudio se enmarca en Puerto Rico, isla del Caribe, específicamente de las Antillas Mayores, y que es un territorio no incorporado de Estados Unidos. Esto la posiciona en un contexto político colonial, una condición que genera múltiples vulnerabilidades, la más notable de las cuales es la sujeción a marcos normativos, visiones y condiciones que no necesariamente se construyen para beneficio de los sujetos colonizados ni de sus espacios. Por otra parte, la isla se encuentra en la ruta de los huracanes del Atlántico, de los cuales Hugo (1989), Georges (1998) y María (2017) están entre los que más la han perjudicado de forma directa e indirecta en los últimos 32 años. También se encuentra en una zona sísmica, por lo que se halla expuesta a los terremotos. 
Estas condiciones, en conjunto, provocan vulnerabilidades que, a su vez, se traducen en la pérdida de vidas, a corto y largo plazo, y de bienes, entre los que la casa es uno de los más preciados.

Comenzar a analizar un contexto posdesastre y sus consecuencias sobre la arquitectura supone múltiples retos. El primero de ellos es tratar de recomponer la imagen del escenario predesastre, aquello que podría considerarse "la normalidad". Si bien el huracán María azotó a Puerto Rico el 20 de septiembre de 2017, el 19 del mismo mes el contexto en el que la isla se encontraba era desalentador $-\mathrm{y}$ no sustancialmente debido al paso del huracán Irma, sino por las múltiples crisis que enfrentaba la isla, entre las que se destacaba la crisis socioeconómica (especialmente, desde 2008)—. Las condiciones en que se desarrollaba lo que en otros análisis podría denominarse "el balance" o "la normalidad" eran todas ellas de inquietante excepcionalidad. Para resumir, ya existía un estado crítico, o crisis múltiples, pues ya experimentábamos una gran alteración o una serie de alteraciones provocadas de formas diversas por el propio ser humano, como la emigración, el desempleo, la depresión económica, el endeudamiento, la pobreza, la desigualdad socioeconómica, la debilitación democrática, la desinformación, la corrupción y la condición de la colonialidad, ahora más exacerbada y siempre influyente.

Estas crisis se han reflejado, a lo largo de la historia, en la calidad y la cantidad de la producción arquitectónica del país, y el siglo XXI no es la excepción: buena parte de esa producción se hallaba en un estado de considerable vulnerabilidad para cuando llegó el huracán María. Algunos de los indicadores o los productos de esta vulnerabilidad son las casas unifamiliares que no son construidas de forma segura, al no tener refuerzos necesarios para resistir los vientos de un huracán, o construidas en zonas inundables o propensas a deslizamientos. Esto último ocurre en diferentes localizaciones en toda la isla, incluyendo áreas montañosas y cerca de la costa, y se exacerbó con las condiciones de desigualdad que provocaron, entre otros fenómenos, la emigración del campo a la ciudad en la década de 1930, cuando las personas comenzaron a ubicar sus viviendas en los terrenos disponibles, pero no necesariamente seguros, cerca de las áreas de trabajo. Por esto, a casi dos años del paso del huracán, ya es un lugar común que afirmen lo siguiente quienes se han dispuesto a analizar críticamente este contexto: por un lado, que a una crisis o un estado de vulnerabilidad ya existente sobrevino otra crisis o estado de vulnerabilidad; que ambos cuadros son más efecto del "artificio" humano que de la naturaleza, como sostiene Steinberg (2006); que el huracán María retiró la gran masa de verdor, y así permitió visibilizar los espacios donde mejor se apreciaban el desastre, la crisis o la vulnerabilidad precedente, y que el capitalismo del desastre (Klein, 2007), históricamente imple- mentado en Puerto Rico, ganaba, en todo caso, nuevos dominios. No obstante lo anterior, poco se ha reflexionado sobre la forma como las condiciones mencionadas tenían resonancia en el estado de la arquitectura y la vivienda, ni sobre cómo ambas podían asistir en la producción y la reproducción, a su vez, de aquellas debilidades y riesgos. El estado de la disciplina y la profesión de la arquitectura no era favorable, ya que desde antes del huracán el número de arquitectos licenciados ${ }^{1}$ y de obras decayó abruptamente, en consonancia con el aumento de la emigración y la más reciente depresión de la actividad económica, desde 2008. Según datos censales, la población de Puerto Rico decreció de 3808610 habitantes en el 2000 a 3725789 en el 2010 . Las personas empleadas como arquitectos disminuyeron de 330 en 2012 a 260 en 2017, según las estadísticas de empleo y salarios por ocupación. En el sector de la vivienda, específicamente, algunos desequilibrios eran más que preocupantes: contábamos con la existencia de 75 000-90 000 unidades de vivienda autoconstruidas $^{2}$, lo que refleja la necesidad de vivienda asequible, mientras que, por otro lado, se sumaban 334564 unidades de vivienda vacantes en Puerto Rico en $2016^{3}$ (368 174 unidades vacantes, según la Encuesta Sobre la Comunidad de 2015 a 2019).

Luego del fenómeno, a juzgar por la documentación fotográfica disponible y ampliamente difundida por los medios, además de la experiencia del huracán (pues, en el momento en que se escriben estas líneas, en Puerto Rico no contamos aún con muchos datos concluyentes ni con suficientes documentos oficiales fiables ni verificables sobre el evento ni sobre sus efectos ${ }^{4}$ ), y en las observaciones de campo realizadas por las autoras se deduce que la vivienda unifamiliar fue, muy posiblemente, una de las tipologías más afectadas/destruidas por el paso de María. Según la Agencia Federal para el Manejo de Emergencias de Estados Unidos (en inglés, FEMA, por las iniciales de Federal Emergency Management Agency), más de 166000 viviendas se encuentran en proceso de reparación o reconstrucción en 2019. De estas, la agencia estadounidense indica que solo 4853 unidades fueron destruidas,

1 Según la definición de la Junta Examinadora de Arquitectos y Arquitectos Paisajistas de Puerto Rico y el Colegio de Arquitectos y Arquitectos Paisajistas de Puerto Rico.

2 Debido a la cantidad de construcciones realizadas sin permiso, determinar cuántos edificios existen según su tipología o su uso es prácticamente imposible. Ver: "En Puerto Rico hay hasta 90.000 casas sin permiso de construcción", El Nuevo Día, 28 de noviembre de 2017.

3 Hay unas 334564 unidades de vivienda vacantes en Puerto Rico, según el Negociado del Censo de Estados Unidos, Encuesta sobre la Comunidad de Puerto Rico de 2012 a 2016.

4 La revista New England Journal of Medicine publicó un estimado de la Universidad de Harvard con 4645 muertes atribuidas al paso del huracán. La infame cifra inicial provista por el gobierno de Puerto Rico fue de 64. La cifra final oficial provista el 28 de agosto de 2018 fue de 2975 muertes, tras haberse comisionado un estudio a la Universidad de Washington. 
considerando destruidas a aquellas que no se pueden habitar como efecto del paso del huracán ${ }^{5}$. Posiblemente por esa razón, es la tipología que parece haber merecido más atención por parte de arquitectos y diseñadores, así como de otros agentes -especialmente, en la primera fase de recuperación-; sin embargo, otras razones para esta atención radican en la importancia que adjudicamos sociológica y culturalmente a la vivienda, al igual que en la visión limitada de lo que comúnmente se contempla como el espacio de acción de nuestros profesionales de la arquitectura, aun autodefinidos en su gran mayoría dentro de prácticas ortodoxas que contemplan la creación de objetos arquitectónicos ex novo como única forma de producción espacial. No obstante, al reflexionar en cómo aportar a la transformación del nuevo escenario, se opta por enfocarse en el discurso desde el que se comenzó a abordar, significar y representar el proceso de recuperación; en particular, el de la vivienda. La resiliencia ha sido otro lugar común en las narrativas del contexto posdesastre, al menos desde la Cumbre Mundial de Desarrollo Sostenible, en Johannesburgo, Sudáfrica (2002), y en Puerto Rico, con notable presencia tanto dentro como fuera del ámbito de la arquitectura, luego del paso del huracán María. Esto despertó suspicacias en las investigadoras y condujo a reclamar una dimensión menos explorada del término.

Lawrence Vale y Thomas Campanella (2005), quienes han teorizado sobre el desastre y la resiliencia de ciudades de la modernidad, observan que luego de un desastre, entre las políticas implicadas en la reconstrucción figuran las políticas de sucesión simbólica y las políticas de procesos institucionales, que se preguntan, respectivamente: ¿Cómo el poder simbólico de lo construido puede usarse para atraer el ataque/ riesgo (cuando se trata de destrucción perpetrada por el ser humano) o ser usado como signo de recuperación? Y, ¿Qué revela el proceso de recuperación sobre el balance de poder que la sociedad busca reconstruir/construir? (Vale \& Campanella, 2005, p. 9). Ambas interrogantes sirven de guía en esta investigación. Respecto a la primera, es posible argumentar que el poder simbólico de una casa resiliente radicaría en su capacidad para aplacar los riesgos reales, para concretar una "recuperación" real y radical, más que en su capacidad para reproducir una imagen de normalidad, pero ocultando una vulnerabilidad. La casa podría ser usada como signo de recuperación, pero ese proceso de "recuperación" interesa, en todo caso, como ocasión para agenciar una transformación definitiva del espacio doméstico, del espacio común y del país. Esta transformación debe gestarse con y desde los habitantes, evitando plantearse, como señala Luz Marie Rodríguez (2012, p. 165), como un ejercicio de "[re]construcción de la identidad",

5 Ver: https://www.fema.gov y sorteando la injerencia de otros intereses sobre Puerto Rico ${ }^{6}$.

En este sentido, Kevin Rozario indica que, históricamente, la narrativa posdesastre tiende a poner al desastre como punto culminante, como centro o punto de inflexión desde el que se inicia una transformación, como una peripecia (2005, p. 33); no obstante, en el contexto colonial de Puerto Rico, como territorio no incorporado de Estados Unidos, sujeto a su poder político, económico y social directo, esta narrativa puede tener una dimensión más que perversa si no se consideran los mecanismos que limitan el campo de acción. No se puede animar a la espera de un desenlace esperanzador si el proceso de recuperación consiste en la reproducción/“recuperación" de una condición y una visión colonial; sin embargo, como han sugerido otros autores (Reghezza-Zitt et al. 2012), el momento posdesastre puede ser interpretado también como un momento intersticial, un "entre medio", un momento o un espacio de discontinuidades, ambivalente. Así entendido, es como este podría ser el lugar desde el que se puedan producir nuevas condiciones (Reghezza-Zitt et al., 2012, p. 8). En el caso que nos ocupa, se confirma la observación de Homi Bhabha: "el poder colonial [y sus efectos en nuestro país] mina su propia autoridad [pero a la vez] provee, paradójicamente, medios y oportunidades para la resistencia" (2002, p. 55). El contexto del Ilamado posdesastre -luego de un evento, exacerbar las condiciones de vulnerabilidad-, entonces, puede reconfigurarse como espacio para la transformación, para la creación de eutopías, o utopías realizables, como las Ilamaba Ernst Bloch (2004), más que solo como espacio para una "vuelta a la normalidad". La reconstrucción de la vivienda, o más específicamente la casa, se puede convertir en una de las oportunidades o en uno de los gestos con los cuales generar dicha resistencia.

\section{Metodología}

La metodología que guía esta reflexión responde a un modelo mixto, por lo que, partiendo de la investigación cualitativa y la argumentación lógica, se integran, además, aspectos de la investigación histórica y el estudio de casos. A través de nuestro análisis cualitativo, analizamos una situación que surge de nuestra contemporaneidad; específicamente, en el complejo contexto puertorriqueño posdesastre, desde la definición de una serie de conceptos teóricos que resumen y enmarcan el interés de las investigadoras en la relación entre la resiliencia, la resistencia y la casa o el espacio de habitar. Este acercamiento permite interconectar la arquitectura y el diseño con las definiciones de conceptos y narrativas del

6 La autora sugiere que la reconstrucción de la identidad puertorriqueña a través del diseño de la arquitectura y la ciudad supone la transculturación o la asimilación de valores estadounidenses. 
desastre (Boano \& Hunter, 2012; Klein, 2007; Rozario, 2005; Steinberg, 2006), la resiliencia (Diprose, 2014; Reghezza-Zitt et al. 2012; Vale \& Campanella, 2005), la resistencia (Bhabha, 2002) y hechos como la colonialidad, la crisis económica de Puerto Rico en 2008 y el desastre ocurrido en 2017. A partir de aquí, la argumentación lógica propone e interconecta una serie de características que son constitutivas de un tipo de diseño arquitectónico capaz de producir o agenciar la resistencia deseada ante los desastres. Tanto la investigación histórica como el estudio de casos sirven para conocer qué aspectos del pasado y del presente arquitectónicos contienen dichas características, y sirven así de precedentes con los cuales hacerlas visibles y demostrar la posibilidad de su materialización.

\section{Resultados}

\section{Repensar la casa en el contexto posdesastre}

Sobre la vivienda en el ámbito conceptual del momento posdesastre, Camilo Boano y William Hunter (2012) advierten que hay que evadir pensar en esta del modo como suelen hacerlo las concepciones más ortodoxas e incompletas. Pensar la vivienda como mero objeto contenedor puede obstaculizar su capacidad transformadora en el contexto posdesastre. Dicen los autores que "La aproximación en dicho contexto ha tendido a la conceptualización de la vivienda solo como un objeto edificado, estandarizado, concebido 'desde arriba', centrado en la tecnología y como producto terminado" 7 (Boano \& Hunter, 2012, p. 4). Y señalan que "No se suelen integrar las prácticas cotidianas, la cultura y las relaciones sociales. En cambio, se debe entender la casa como un espacio y lugar significativo ya no dividido, entre casa-estructura o casa-constructo social" (p. 3).

Esta, precisamente, suele ser la diferencia más relevante entre la casa de emergencia, que cumple con un propósito básico de proveer refugio o albergue inmediato (en el sentido más técnico o práctico), y el tipo de vivienda que pretenderíamos ver producirse en lo que podríamos llamar la recuperación definitiva o la próxima normalidad. La vivienda de emergencia asiste o es un paso en la recuperación, pero no necesariamente la consideramos un ejercicio conducente a una recuperación definitiva. En el mejor de los casos, lo que se edifica de forma rápida ante la emergencia podría resignificarse como un modelo transicional o como origen de una construcción a largo plazo. Un ejemplo de esto es RAPIDO Recovery ${ }^{8}$, un modelo implementado en Texas, Estados Unidos, luego de que el huracán Dolly azotó el Golfo de Texas en 2008.

7 Traducción de las autoras.

8 http://www.rapidorecovery.org
Así, la casa, como la entendemos en su carácter permanente o con su intención de permanencia, debe integrar el concepto de domesticidad que Witold Rybczynski (1986) define como "un conjunto de emociones percibidas, no solo un atributo aislado [que] incorpora sentimientos [de familiaridad, intimidad, etc.], y no solo les da refugio" (p. 84). Si la casa no tiene éxito incorporando esta domesticidad, como advierte Juhani Pallasmaa (1995), esta puede reificar la miseria humana. Para evitarlo, esta debe superar su función como contenedor que precariamente protege y que reúne en su diseño conceptos de forma y proporciones (entre otros), y albergar vivencias que se acumulan en el tiempo, para convertirse así en un hogar. Los atributos de un hogar superan de esta manera los límites de los comúnmente reconocidos como componentes o elementos de composición arquitectónica. Esta es una diferencia que también destaca Amos Rapoport (2000, p. 148), cuando explica, respectivamente, el seleccionismo y el instruccionismo de la vivienda vernácula y la vivienda estilizada y reproducida en la contemporaneidad.

El diseño de una vivienda puede ser guiado, además, por "signos de ocupación" (Smithson \& Smithson, 1972, p. 97) y la observación del "lenguaje de patrones" de un lugar. Según Christopher Alexander (1981, p.11), existe una relación entre el carácter de un lugar y los acontecimientos que ocurren en él; por tanto, la manera como las personas utilizan o transitan un espacio, corresponde a los atributos de este espacio, y viceversa. Incorporar los patrones de un lugar en el diseño de las viviendas que allí se sitúen contribuye a que los habitantes puedan sentirse familiarizados con el espacio doméstico y puedan convertirlo, sin inconvenientes, en su hogar. En la transformación del espacio, habitar y construir, tal como describe Heidegger (1994), son actos que no se desligan. El concepto de cultura o las relaciones sociales entre las personas y el contexto tampoco debe desatenderse. Según Rapoport (1972, p. 83), es importante resaltar también lo que caracteriza a un grupo; es decir, cómo este grupo elige protegerse de lo adverso y cómo expresa su filosofía de vida.

\section{Una casa resiliente/resistente}

La resiliencia es otro de los lugares comunes del análisis posdesastre, como ya se ha subrayado. Fácilmente, se lo presenta y se lo pretende como concepto opuesto a la resistencia, pero, como parte de esta investigación, se la aborda como una de sus vías o sus eslabones. Es un concepto que ha ido ganando presencia a través de los años y ante contextos de desastre. Algunos autores han apuntado que, ante la supuesta incapacidad técnica para resistir la amenaza, se opta por adaptarse a esta, y no cuestionarla (Reghezza et al., 2012). Otros, como Kristina Diprose (2014), explican que la resiliencia sería fútil si esta fuera considerada respuesta, tolerancia ante la 


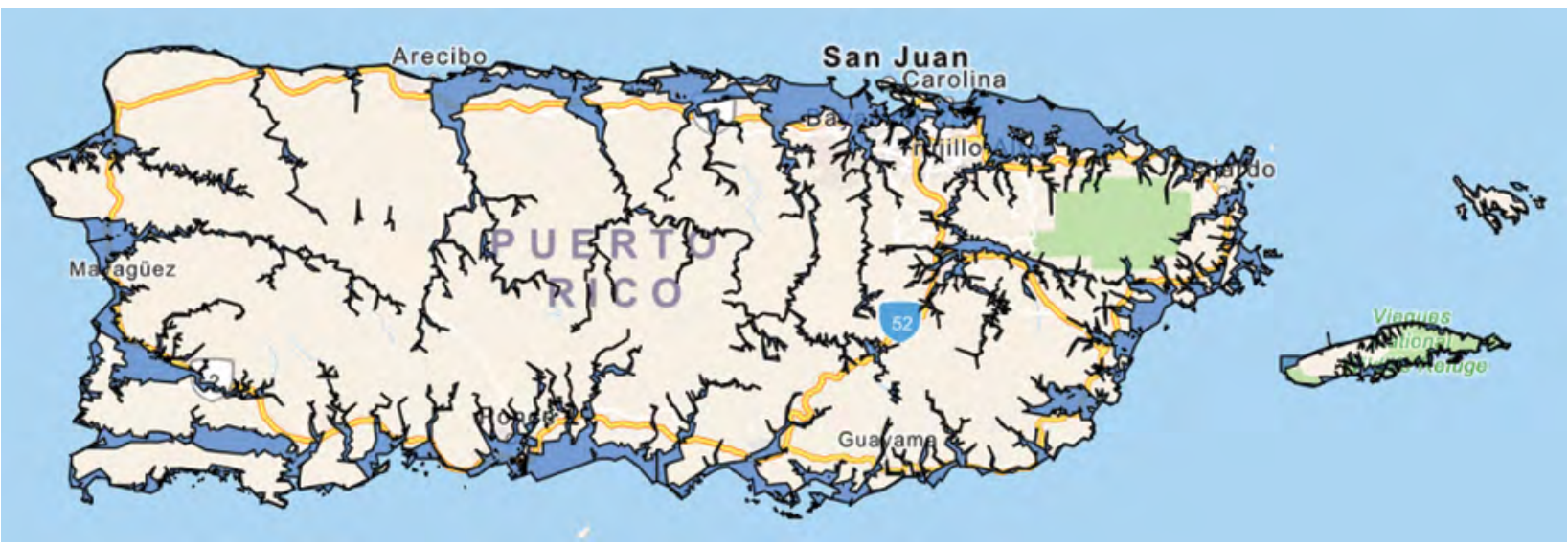

A Figura 1. Mapas de zonas inundables en Puerto Rico.

Fuente: ArcGis y estuario.org austeridad y la pobreza, aspiración del comportamiento humano. Así entendida, la resiliencia supondría la aceptación pasiva y la reproducción de las condiciones de vulnerabilidad, inequidad e injusticia, bajo la promesa de cambios o tecnologías venideros. La autora advierte que la resiliencia, como concepto, puede servir para desarmar discursivamente la posibilidad de articular cambios profundos y reales en contextos de vulnerabilidad, y en el caso de Puerto Rico, así ha comenzado a servir al discurso oficial.

No obstante lo anterior, esta investigación plantea que la resiliencia puede posibilitar también la creación de esos cambios profundos, y la define, de forma ambivalente, como propiedad y capacidad, como característica y potencialidad: propiedad de resistir y capacidad para anticipar los efectos de vulnerabilidades y riesgos reales o posibles, y responder a estos; incluso, asistiendo a su vencimiento. En este último caso, se trata de una propiedad/capacidad atribuible a una vivienda, cuyos riesgos provienen de al menos dos fuentes distintas, pero interconectadas: el contexto geográfico, en el que son frecuentes huracanes y terremotos, y el contexto socioeconómico y político particular, de crisis y vulnerabilidad. Ante el primer escenario, la casa resiliente anticipa los efectos de estos eventos y responde a ellos, es contextualizada, mientras que en el segundo escenario la casa pretende transformar o vencer las limitaciones de su contexto. Se convierte en un mecanismo para afrontar los eventos naturales, pero también, para resistir a los efectos de la agencia humana, incluyendo la transformación del medio ambiente. Así, la casa resiliente sería un espacio de ambivalencia-que-genera-resistencia.

\section{Cómo posibilitar la resistencia a partir de la resiliencia}

La casa resiliente (sea unifamiliar, colectiva o covivienda) logra oponerse a los eventos naturales y los contextos de crisis/vulnerabilidad mediante parámetros de diseño y codiseño, que atiende por grados. Las siguientes características, en conjunto, son las que, a partir de la observación y el análisis de lo ocurrido, podrían entenderse como las que adelantarían el hecho de solventar las carencias visibles en las viviendas del país. Estas son la localización y que la casa sea sostenible, asequible, accesible, adaptable, flexible, incremental o, como resultado de una reutilización adaptativa, producida mediante un proceso de diseño participativo, autoconstruida o modular. Ninguno de los ejemplos que se citan a continuación reúne todas las características, pero todos aportan a la noción de que la casa resiliente es realizable.

En primer lugar, la casa resiliente debe tener una localización apta. Su diseño o su rediseño comienzan con la elección o la concienciación de sus gestores y de sus diseñadores ante el emplazamiento, considerando aspectos determinantes como la topografía y las cotas de inundabilidad. Históricamente, las comunidades más afectadas por el paso de los huracanes son las que habitan zonas inundables o en condiciones geológicas, topográficas o del suelo que resultan inadecuadas o precarias. En la figura 1 se muestran las zonas inundables en Puerto Rico y en el Estuario de la Bahía de San Juan. Puede verse que en su mayoría están cerca de la costa, ríos o canales como el Caño Martín Peña.

Las personas habitan estas zonas debido a múltiples razones que incluyen, aunque no se limitan a ello, los precios del suelo y la vivienda y la insensible repartición gubernamental de parcelas y lotes. Luego del huracán María, por otra parte, fue más evidente que dentro de los atributos positivos del emplazamiento figura su identificación como espacio de una comunidad. La densidad poblacional con matices generacionales y culturales, comercios y servicios supone una red de apoyo que permite que los habitantes del lugar se enfrenten mejor a la amenaza. La etnóloga Ivette Chiclana (2011) afirma que en Puerto Rico "La familia extendida y los lazos de compadrinazgo son los mecanismos socio-políticos por excelencia del arrabal, pues suplían tanto para las necesidades económicas como para las emocionales" (p. 233).

En segundo lugar, la casa resiliente tiene que ser sostenible y ajustarse a principios ecológicos. Con el uso de tecnología pasiva, ya presente en la arquitectura vernácula puertorriqueña, la casa debe aprovechar los recursos naturales que pueden servirles de sistemas de aclimatación, almacenamiento de agua, iluminación y energización. 


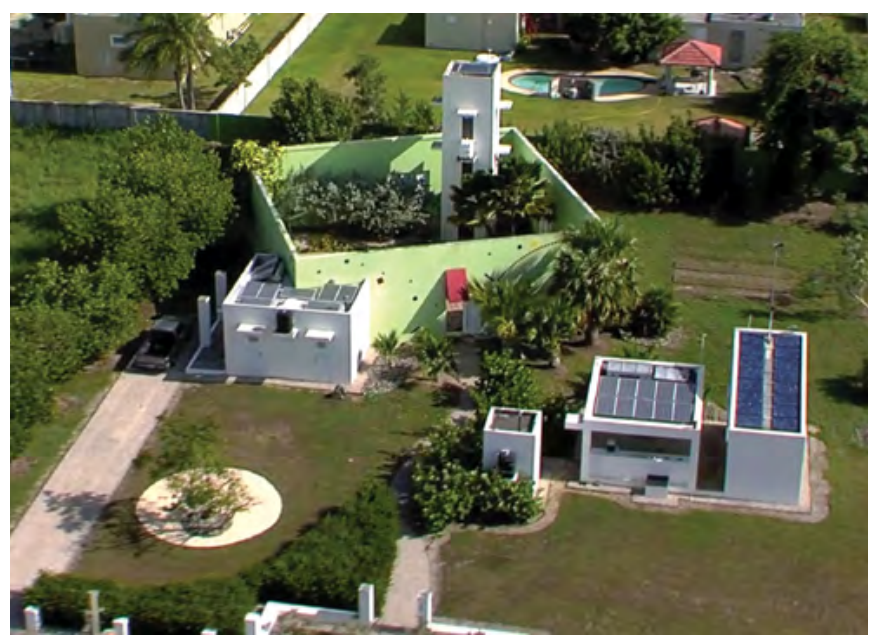

A Figura 2. Casa Ausente.

Fuente: Fernando Abruña (1999) (C Copyright).

De este modo, la casa también instauraría el pensamiento relacional que, según los preceptos de Arturo Escobar (2016), se genera en el habitante y lo obliga a pensarse integrado, y no ajeno, a un contexto tan abarcador como el territorio/país/ mundo. La casa tiene que responder al comportamiento habitual o predecible de los fenómenos naturales o atmosféricos y sus efectos. En Puerto Rico, tan solo la pérdida de la energía eléctrica por un periodo prolongado luego del paso del huracán María provocó más decesos que las inundaciones o los deslizamientos de tierra9 ${ }^{9}$. Tener en funcionamiento un aparato como una máquina de diálisis o de terapia respiratoria puede marcar la diferencia entre la vida y la muerte. Asimismo, el acceso al agua potable es una necesidad vital. El trabajo del arquitecto Fernando Abruña es un precedente relevante, ya que este ha promovido la construcción de vivienda sostenible y autosuficiente, y cuya Casa Ausente, que se muestra en la figura 2, fue el comienzo de una intensa exploración en 1999. Tras el paso del huracán María, Abruña diseñó el modelo de Casa Rescate, que se ve en la figura 3, y la Casa Enlace Resiliente, mostrada en la figura 4, para un concurso convocado por el Colegio de Arquitectos y Arquitectos Paisajistas de Puerto Rico.

Asimismo, la firma Marvel Architects lideró la instalación de paneles fotovoltaicos en varios centros comunitarios y edificios institucionales en diferentes pueblos de la isla. A esta iniciativa se la llamó Resilient Power Puerto Rico. Luego diseñó el Affordable Single-Family Home Prototype y la CORE house (por las iniciales en inglés de Centrally Organized REsilient House), con cualidades como: funcionar independiente de redes eléctricas, incluir sistemas pasivos de ahorro de energía, tener un interior modular que permite que sus habitantes las adapten a sus propias necesidades y ser construidas con materiales disponibles en el entorno local.

9 Según Omayra Sosa Pascual (2017), "Desde el 20 de septiembre [...] la tasa promedio de muertes diarias aumentó en un $43 \%$ con picos de cerca de un $80 \%$ en días como el 21 y el 25 de septiembre. En octubre, las muertes aumentaron un 23,3\%".

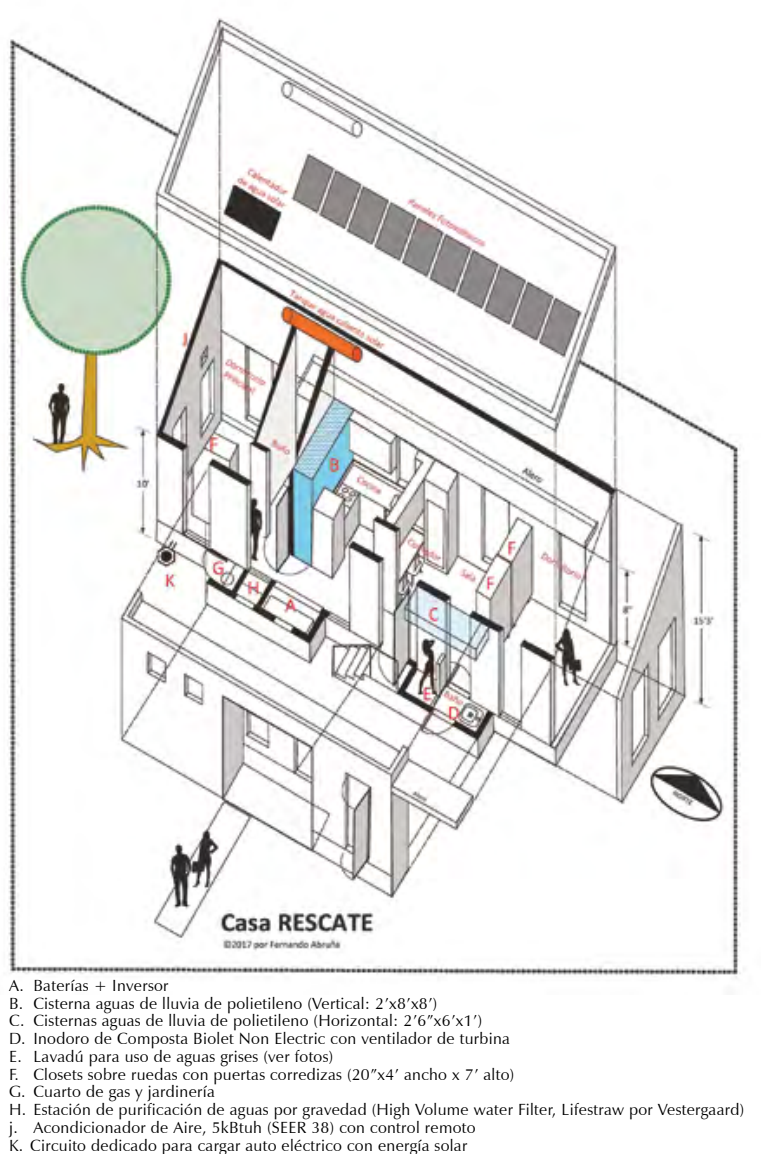

Fernando Abruña (C) Copyright).

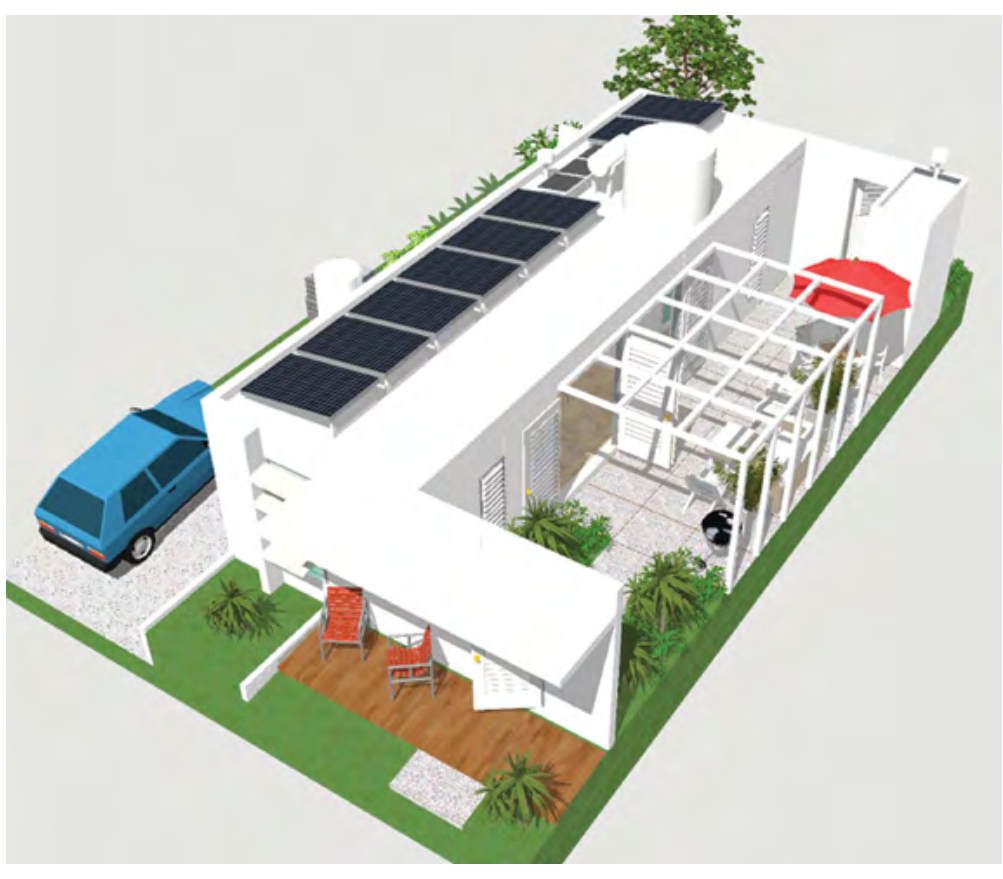

En tercer lugar, la casa resiliente tiene que ser asequible, ser compatible con el costo de vida del grupo social mayoritario al que se destina, en lugar de ser un instrumento de endeudamiento que provoque, entre otros efectos, la menor calidad constructiva. Una forma de lograrlo es mediante la revisión y el análisis de los procesos constructivos y las tecnologías disponibles, la investigación de materiales, la consideración de nuevas formas de tenencia, etc. De este modo, la casa comprende y sortea las restricciones del contexto/sistema socioeconómico. Tener un techo seguro es un derecho, que deben reconocer tanto diseñadores como gestores y Estado (proveyendo, en su caso, los mecanismos necesarios para la construcción de vivienda social). 
Como cuarto aspecto, la casa resiliente tiene que ser accesible, tiene que incorporar principios del diseño universal, por cuanto anticipa una población variable. La accesibilidad no se limita a personas con movilidad reducida o diversidad funcional, sino que también aplica a los adultos mayores, cuyos movimientos son cada vez más limitados; es decir, a la larga aplica a todos. En el caso de los países con una población cada vez más envejecida, se hace más evidente la pertinencia de este parámetro, y esta no se pierde al examinar la heterogeneidad de los hogares.

En quinto lugar, la casa resiliente individual debe ser adaptable y flexible, bien ante condiciones naturales y condiciones tanto sociales como físicas; es decir, sin jerarquías que fuercen usos predeterminados o que impidan su transformación. La adaptabilidad, considerando las definiciones de Jeremy Till y Tatjana Schneider (2007), implica que una casa se pueda habitar de distintas maneras, sin necesidad de que se realicen en ella grandes cambios, como la remoción o la construcción de nuevas paredes; sin embargo, la flexibilidad implica que una vivienda podría tener paredes móviles, mobiliario transformable o, simplemente, ser un soporte fijo con una unidad separable interior/intermedia que se puede organizar de diversas maneras, o cambiar de forma sencilla a través del tiempo, tal como lo describía el arquitecto John Habraken (2000, p. 18). Tanto las colonias Guerrero y Tepito, diseñadas por el arquitecto mexicano Jorge Andrade, como las medias viviendas, diseñadas por el arquitec-

$\rightarrow$ Figura 5. Proyecto de soportes y unidades separables de vivienda colectiva, diseñado por el arquitecto Jan Wampler en 1967.

Fuente: Jan Wampler (1967) (C) Copyright).
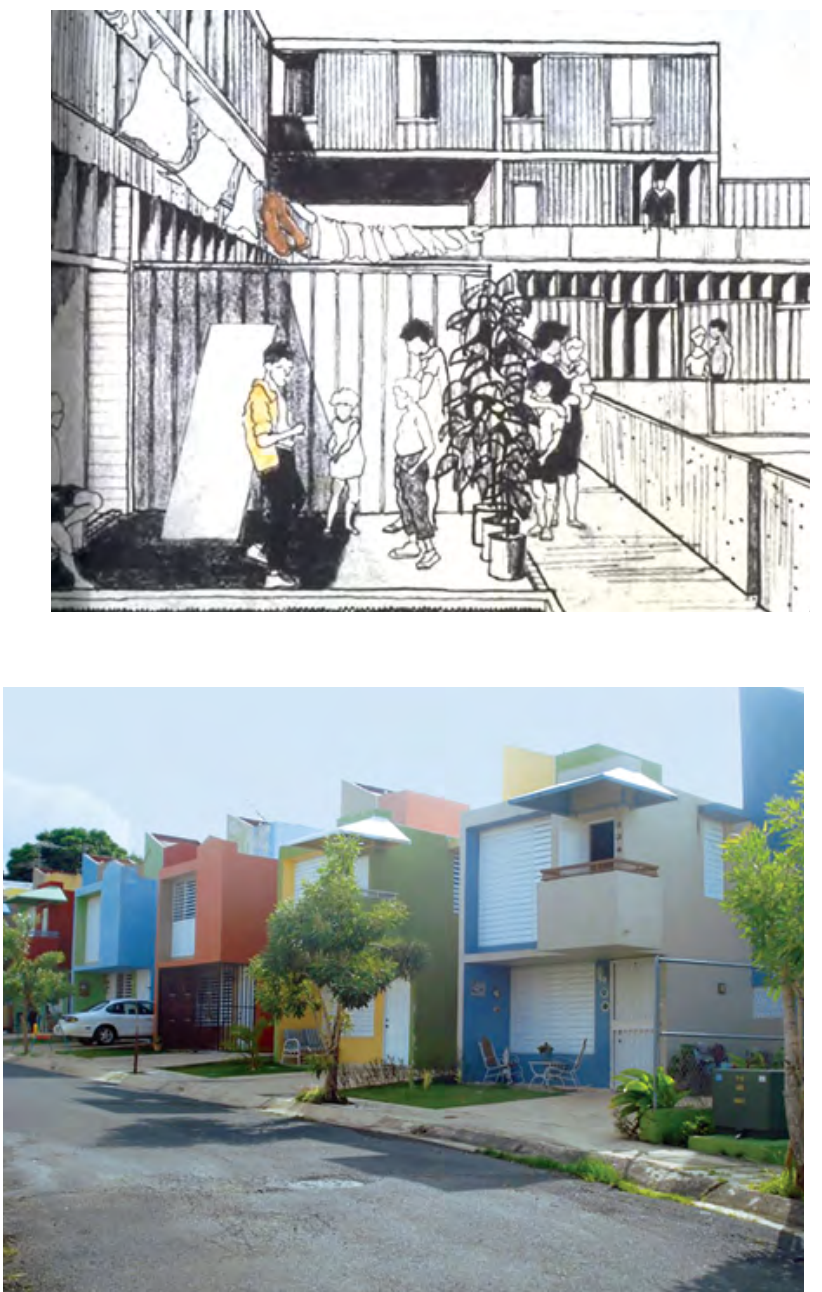

to chileno Alejandro Aravena, son ejemplos de soportes fijos con unidades separables.

En Puerto Rico, a pesar de que el modelo de la vivienda colectiva fortalece lazos de solidaridad, como ya se mencionó, solo se ha explorado su flexibilidad a modo de soportes y unidades separables, según el concepto de Habraken, por el arquitecto Jan Wampler en $1967^{10}$, como se observa en la figura 5 .

La casa individual o en hilera también puede ser incremental, según como aumenten sus usos, o los sujetos o los núcleos familiares que las habitan. El arquitecto Edwin Quiles, consciente del modo de habitar de las familias puertorriqueñas y su necesidad de "mejorar" sus viviendas con el tiempo (típicamente, ampliándolas), exploró el diseño de vivienda incremental en el barrio de Juan Domingo, la Nueva Trujillo, como se muestra en la figura 6, en el pueblo de Guaynabo, en 2002, y luego del huracán María, diseñó la Casa Semilla respondiendo al mismo parámetro, ilustrado en la figura 7. La incrementalidad, incluso, proveería la ocasión de transformar la vivienda individual en colectiva.

Las cualidades de adaptabilidad, flexibilidad e incrementalidad transcienden la necesidad de los habitantes de personalizar el espacio doméstico. Parte de las viviendas que sufrieron daños en Puerto Rico luego del huracán María eran adiciones en segundas plantas para alojar a hijos que se emancipan, o bien, a nietos o abuelos cuyos ingresos no les permiten adquirir una vivienda nueva en otra localización. Otra razón para ello es que desean o requieren estar cerca de su familia porque necesitan atención especializada, como suele ser el caso de las personas de la tercera edad. También, algunas personas construyen adiciones porque crean un espacio comercial en la primera planta o porque alquilan la primera o la segunda plantas. En ocasiones, estas adiciones son precarias, y por eso sufren daños. Esto se puede constatar, por ejemplo, en las comunidades aledañas al caño Martín Peña, en San Juan, donde aproximadamente el $65 \%$ de los techos que se reconstruyeron luego del huracán María fueron de casas en segundas plantas ${ }^{11}$. Es necesario prever esta incrementalidad para sentar las pautas de una construcción segura.

La casa resiliente también podría surgir como resultado de una reutilización adaptativa de una estructura preexistente. En este caso, la estructura preexistente serviría de soporte, ya haya sido construida con el fin de ser vivienda o para otros usos. En el contexto de zonas urbanizadas con cantidades considerables de edificios vacíos, la reutilización adaptativa funciona como estrategia

10 Wampler diseñó viviendas en La Puntilla (zona periférica del Viejo San Juan) para relocalizar a los residentes del barrio de La Perla, aunque el proyecto nunca se construyó.

11 Según datos de las gerentes de proyecto Omayra Rivera y María Gabriela Flores y entrevista al constructor de Techos para el Caño, Michael Ortíz, de Muscle Builders. 


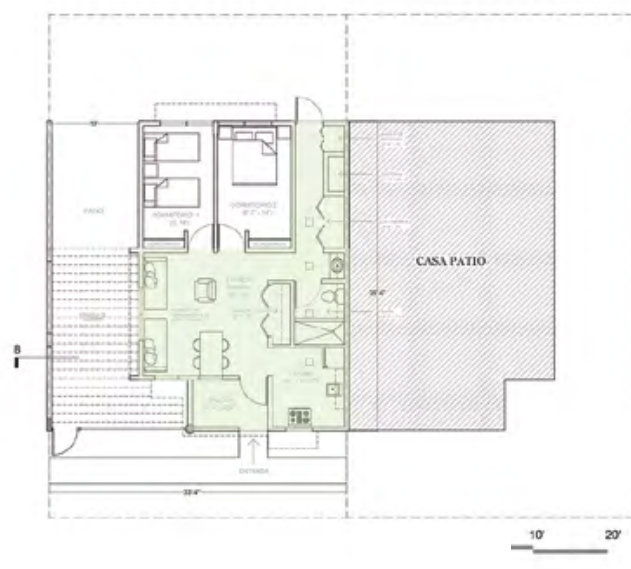

Planta de Piso Escala: $1 / 8^{\prime \prime}=1^{\prime}$

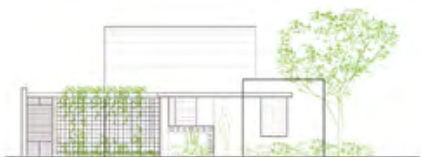

Elevación Lateral Escala: $1 / 8^{\prime \prime}=1^{\prime}$

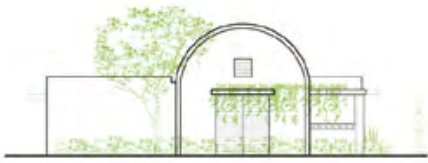

Elevación Trasera Escala: $1 / 8^{\prime \prime}=1^{\prime}$

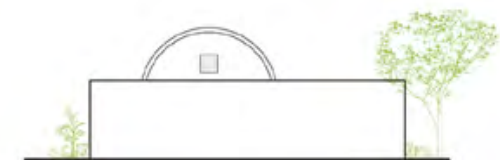

Elevación Lateral Escala: $1 / 8^{\prime \prime}=1^{\prime}$

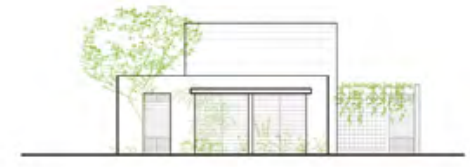

Elevación Frontal Escala: $1 / 8^{\prime \prime}=1^{\prime}$

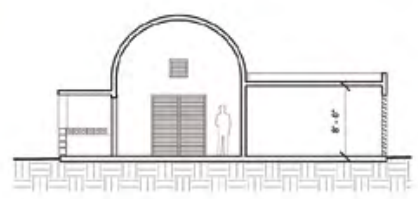

Corte A-A Escala: $1 / 8^{\prime \prime}=1^{\prime}$

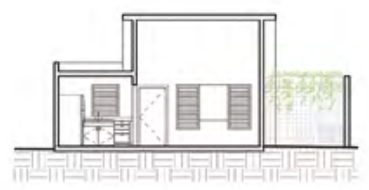

Corte B-B

Escala: $1 / 8^{\prime \prime}=1^{\prime}$ adicional para combatir los problemas de abandono y deterioro urbano.

Como sexto punto, la casa resiliente debe ser producida mediante un proceso de diseño participativo que incluya de manera activa a sus futuros moradores en el proceso de ideación, composición, proyección, e incluso, en su construcción. En este proceso, se debe evadir a toda costa el uso ideológico del proceso participativo, mediante el cual se produce un simulacro de participación con el único objetivo de apaciguar las probables animadversiones respecto a la obra proyectada, como advierten Boano y Hunter (2012). La participación implica un diálogo y una reflexión tomando como punto de partida el bien común y el entorno natural y en el construido. Es un proceso de educación democrática, tal como lo define el educador Paulo Freire (1994). Este proceso debe estar acompañado de una metodología que, mediante estrategias de visualización, facilite el intercambio de información, por ejemplo, a través de dibujos tridimensionales, maquetas, fotomontajes o collages. Estos pueden incorporar elementos de interactividad que posibiliten la exploración de diversas posibilidades de diseño en un ambiente colaborativo. Los procesos participativos, además, naturalizarían en los usuarios su reconocimiento como agentes y actores relevantes en la definición de sus espacios y de su vida en comunidad.

En caso de tratarse de una vivienda individual, la casa resiliente, además, puede ser autoconstruida. Así, puede construirse por cualquier spatial agent, según la definición de Awan, Schneider y Till (2011); es decir, cualquier agente que tenga en su haber la creación de espacio - sea un arquitecto o no-, mediante gestión participativa o con información pertinente a la mano. En este sentido, la casa resiliente es una casa informada, que aprovecha el conocimiento que contienen tanto las guías profesionales como las metodologías tradicionales o autóctonas. La cualidad de la autoconstrucción (que suele ser descalificada como "informal") es una de las instancias en las que disiente explícitamente de las recomendaciones oficiales, porque supone la redefinición del arquitecto y de sus funciones o sus obligaciones profesionales/sociales.

En este caso, el arquitecto puede ser también un facilitador que promueva el acceso a la información esbozada a partir de su conocimiento, información que debe adaptarse a la experiencia de los habitantes. Entre los precedentes más conocidos figuran los arquitectos Walter Gropius y Jean Prouvé, quienes — desde mediados de la década de 1920- exploraron con técnicas de construcción con componentes prefabricados. En Puerto Rico, existió el programa de Ayuda Mutua y Esfuerzo Propio, en la década de 1960, en el que se les ofrecía adiestramiento y materiales a los habitantes para que pudieran construir sus propias viviendas; actualmente, existe la iniciativa de Habitat for Humanity, que funciona de la misma manera. También se creó una guía de construcción segura en 1989, luego del paso del huracán Hugo, con el propósito de que la autoconstrucción de vivienda pudiera ser informada. El programa de Ayuda Mutua y Esfuerzo Propio tuvo mucho éxito, aunque perdió fuerza en la década de 1970, y fue eliminado definitivamente en 1996. Hábitat for Humanity continúa vigente, y actualmente ofrece adiestramiento sobre construcción segura. La guía de 1989 debe ser actualizada, y lo más cercano que existe a esto en el presente, es la guía Manténgase Seguro (Keep Safe), de la organización sin fines de lucro Enterprise Community, y publicada en 2019. Paralelamente, los arquitectos Oscar Marty y, más recientemente, Nataniel Fuster, han explorado métodos de construcción con componentes prefabricados de hormigón que podrían facilitar la autoconstrucción formal de viviendas.

Por último, si se tratara de una vivienda individual, la casa resiliente debe, igualmente, ser modular, mediante la incorporación de prefabricados y la utilización de estándares. De este modo, anticipa la naturaleza múltiple de los autores que podría tener, y la necesidad de construirse en un término de tiempo tolerable. Un método de construcción estandarizado proveería una mejor autogestión constructiva y contribuiría al inaplazable abaratamiento de costos. En la década de 1970, en Cuba se exploró con componentes
(A) Figura 7. Casa Semilla (2018). Fuente: Edwin Quiles (2018) (C) Copyright). 


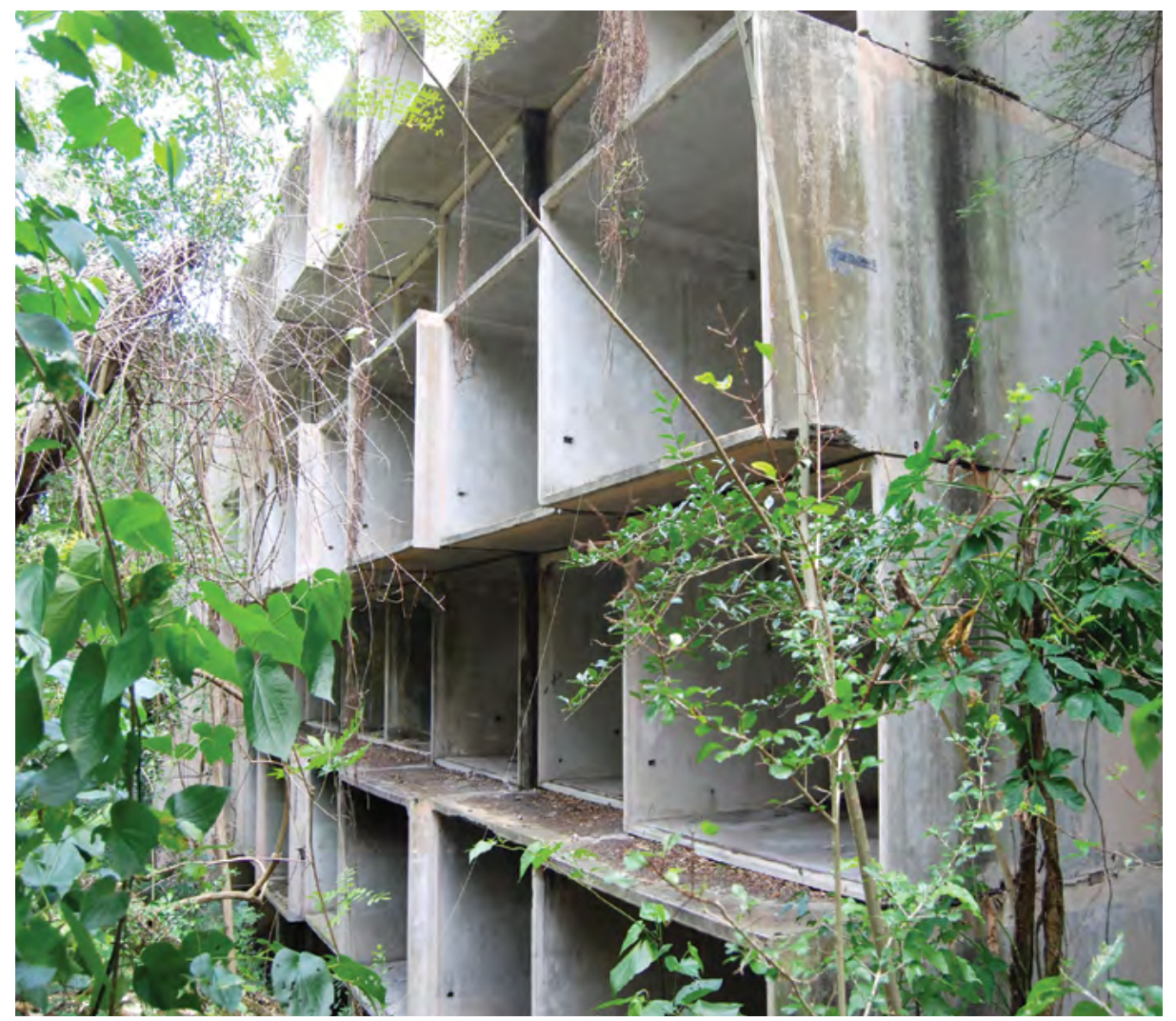

(A) Figura 8. Módulos de Hábitat, según el diseño del arquitecto Moshe Safdie, abandonados en San Juan, Puerto Rico.

Fuente: elaboración propia (2018) (@ Copyright).

$\rightarrow$ Figura 9. Proyecto del estudiante de arquitectura Jorge Acevedo (2018), donde se explora la reutilización adaptativa de los módulos de Moshe Safdie como modelos de vivienda adaptable.

Fuente: Jorge Acevedo (2018) (C) Copyright).

$\rightarrow$ Figuras 10. Proyecto de la estudiante de arquitectura Sachely Feliciano (2018), donde se explora la reutilización adaptativa de los módulos de Moshe Safdie como modelos de vivienda adaptable.

Fuente: Sachely Feliciano (2018 (C) Copyright).

$\rightarrow$ Figura 11. Proyecto de la estudiante de arquitectura Gisela Salas (2018), donde se explora la reutilización adaptativa de los módulos de Moshe Safdie como modelos de vivienda adaptable. Fuente: Gisela Salas (2018) (c) Copyright).
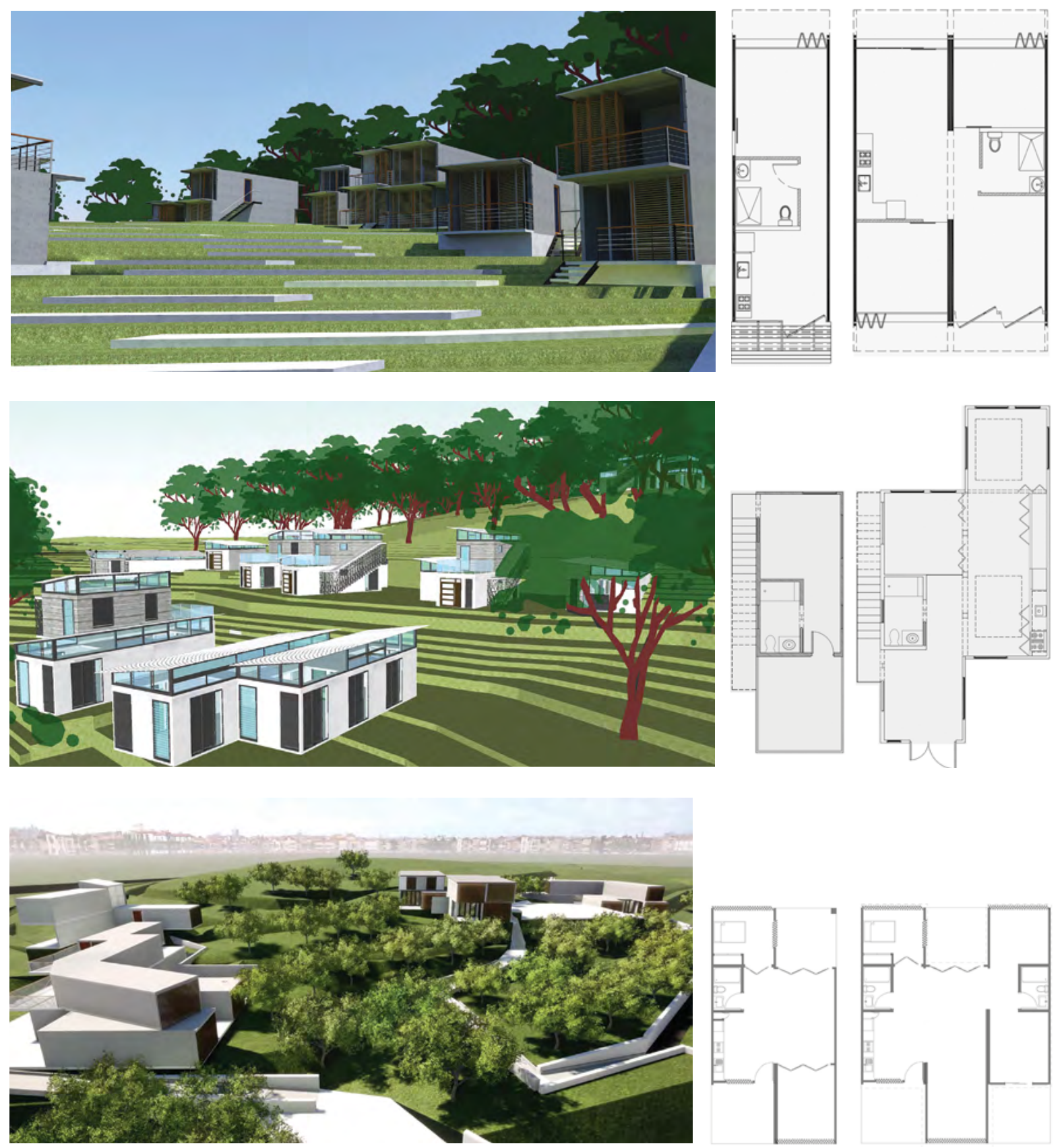


\section{Conclusiones}

En estas cualidades en las que se fundamenta la resiliencia de la casa, o vivienda resiliente, en Puerto Rico (localización segura, sostenibilidad, asequibilidad, accesibilidad, adaptabilidad, flexibilidad, incrementalidad, reutilización adaptativa, producción mediante un proceso de diseño participativo, autoconstrucción o modularidad), no solo se anticipan los problemas que las crisis prevalecientes provocan. Los riesgos a los que natural o socioeconómicamente está expuesto el país, así como sus efectos, pueden ser contrarrestados con diversos grados de contundencia. En el caso de los primeros, ya es posible contar con el conocimiento para minimizar sus efectos, aun cuando serán eventos que seguirán ocurriendo. En el caso de los segundos riesgos, existen estrategias que sirven para resistir a estos, y que pueden provenir, incluso, de la experiencia de repensar, habitar o construir la casa resiliente, como la validación de la experiencia y el conocimiento del pasado, la participación, la democratización del ejercicio creativo o la concienciación de nuestra conexión con el ambiente natural, entre otros. Establecer unos parámetros, comprendiendo el acto de habitar en todas sus dimensiones, posibilita ampliar la gama de conocimiento en relación con la vivienda y explorar soluciones que trascienden el estado de emergencia. Asimismo, el espacio doméstico debe ser visto, junto al acto de habitar, como un proceso, y no como un producto. La casa resiliente podría ser una arquitec-

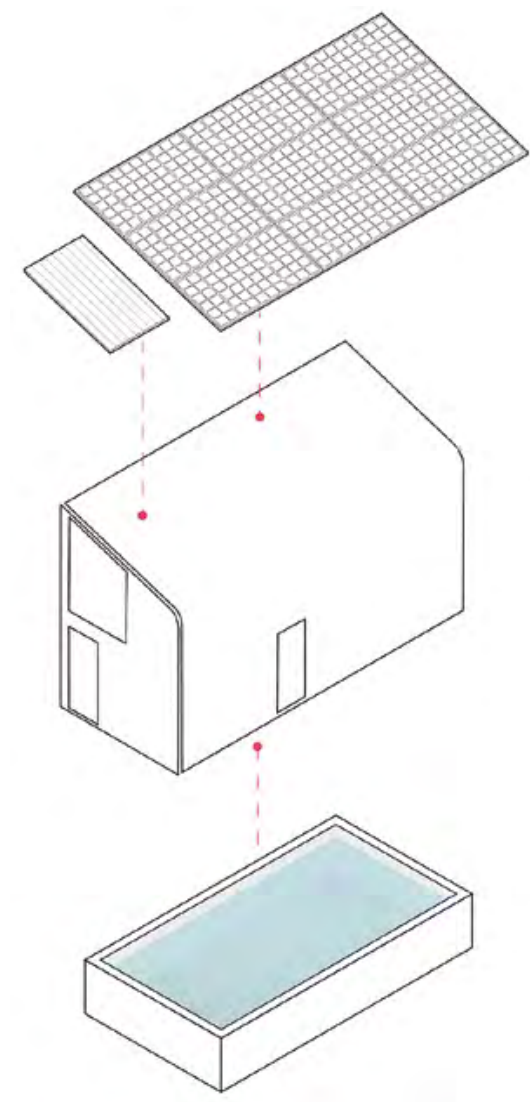

Figura 12. Módulo de la casa-i (2018), diseñada por el arquitecto Nataniel Fuster. Fuente: Nataniel Fuster (2018) (C Copyright).

\section{Referencias}

Alexander, C. (1981). El modo intemporal de construir. Editorial Gustavo Gili. (Documento original: The timeless way of building. New York: Oxford University Press, publicado en 1979).

Awan, N., Schneider, T., \& Till, J. (2011). Spatial agency: Other ways of doing architecture. Routledge.

Bhabha, H. (2002). El lugar de la cultura. Ediciones Manantial. (Documento original publicado en 1994)

Bloch, E. (2004). El principio esperanza. Trotta.

Boano, C., \& Hunter, W. (2012). Architecture at Risk (?): The Ambivalent Nature of Post-Disaster Practice. Architectoni.Ca, 1(1),1-13.

Chiclana, I. (2011. Asomo de una memoria otra: un arenal que se hace barrio, un empeño obrero que se hace comunidad. En J. Lizardi Pollock \& M. Schwegmann (Eds.), Espacios ambivalentes: historias y olvidos en la arquitectura social contemporánea (pp. 229-244). Ediciones Callejón.

Diprose, K. (2014). Resilience is futile: The cultivation of resilience is not an answer to austerity and poverty. Soundings: A journal of politics and culture, 58, 44-56.

https://www.muse.jhu.edu/article/565757.

Escobar, A. (2016). Autonomía y diseño: la realización de lo comunal. Editorial Universidad del Cauca.

Freire, P. (1994). Educación y participación comunitaria. En Nuevas perspectivas críticas en educación (pp. 83-96). Paidós.
Habraken, J. (2000). El diseño de soportes (2a ed.). Editorial Gustavo Gili. (Documento original publicado en 1974).

Heidegger, M. (1994). Construir, habitar, pensar [Conferencias y artículos]. Serbal (documento original publicado en 1954).

Klein, N. (2007). La doctrina del shock. El auge del capitalismo del desastre. Ediciones Paidós.

Pallasmaa, J. (1995). Identity, intimacy and domicile - notes on the phenomenology of home. En N. D. Benjamin (Ed.), The Home; Words, Interpretations, Meanings and Environments (pp. 131-147). Avebury.

Rapoport, A. (1972). Vivienda y cultura. Editorial Gustavo Gili. (Documento original publicado en 1969).

Rapoport, A. (2000). Theory, culture and housing. Housing, Theory and Society, 17(4), 145-165. https://doi. org/10.1080/140360900300108573

Reghezza-Zitt, M., Rufat, S., Djament-Tran, G., \& Le Blanc et Serge Lhomme, A. (2012). What resilience is not: Uses and abuses? Cybergeo: European Journal of Geography, 621. https://doi.org/10.4000/cybergeo.25554

Rodríguez, L. M. (2012). [Re]visión de la vivienda social en San Juan: Notas de la arquitectura para el obrero. En J. Lizardi Pollock \& M. Schwegmann, Espacios Ambivalentes: historias y olvidos en la arquitectura social contemporánea (pp. 156-184). Ediciones Callejón.
Rozario, K. (2005). Making progress: Disaster narratives and the art of optimism in Modern America. En L. J. Vale \& T. J. Campanella, The resilient city: How modern cities recover from disaster. Oxford University Press.

https://oxford.universitypressscholarship.com/ view/10.1093/oso/9780195175844.001.0001/ isbn-9780195175844-book-part-6

Rybczynski, W. (1986). La casa: historia de una idea. Editorial Nerea.

Till, J., \& Schneider, T. (2007). Flexible housing. Architectural Press.

Smithson, A., \& Smithson, P. (1972). Signs of occupancy. Architectural Design,(2).https:// www.pidgeondigital.com/talks/signs-ofoccupancy/

Steinberg, T. (2006). Acts of God: The unnatural history of disaster in America. Oxford University Press.

Sosa Pascual, O. (2017, 7 de diciembre). Se disparan en casi mil las muertes tras María. CPI Centro de Periodismo Investigativo. https://periodismoinvestigativo. com/2017/12/se-disparan-en-casi-mil-lasmuertes-tras-maria/

Vale, L. J., \& Campanella, T. J (Eds.). (2005). The cities rise again, in the resilient city: How modern cities recover from disaster. Oxford University. 

(1) Portada: Las bovedadas de Bramante.

Fotografía: Luis Alberto Martínez Camacho (2021) CC BY-NC

\section{(ब) (1) (\$)}

(A) Orientación editorial

\section{Enfoque y alcance}

La Revista de Arquitectura (Bogotá) ( (ISSN 1657-0308 Impresa y E-ISSN 2357-626X en línea) es una publicación científica seriada de acceso abierto, arbitrada mediante revisión por pares (doble ciego) e indexada, en donde se publican resultados de investigación originales e inéditos.

Está dirigida a la comunidad académica y profesional de las áreas afines a la disciplina. Es editada por la Facultad de Diseño y el Centro de Investigaciones (CIFAR) de la Universidad Católica de Colombia en Bogotá (Colombia).

La principal área científica a la que se adscribe la Revista de Arquitectura (Bogotá) según la OCDE es:

Gran área: 6. Humanidades

Área: 6.D. Arte

Disciplina: 6D07. Arquitectura y Urbanismo

También se publican artículos de las disciplinas como 2A02, Ingeniería arquitectónica; 5C03, Estudios urbanos (planificación y desarrollo); 6D07, Diseño.

Los objetivos de la Revista de Arquitectura (Bogotá) son:

- Promover la divulgación y difusión del conocimiento generado a nivel local, nacional e internacional

- Conformar un espacio para la construcción de comunidades académicas y la discusión en torno a las secciones definidas.

- Fomentar la diversidad institucional y geográfica de los autores que participan en la publicación.

- Potenciar la discusión de experiencias e intercambios científicos entre investigadores y profesionales.

- Contribuir a la visión integral de la arquitectura, por medio de la concurrencia y articulación de las secciones mediante la publicación de artículos de calidad.

- Publicar artículos originales e inéditos que han pasado por revisión de pares, para asegurar que se cumplen las normas éticas, de calidad, validez científica, editorial e investigativa.

- Fomentar la divulgación de las investigaciones y actividades desarrolladas en la Universidad Católica de Colombia.
Palabras clave de la Revista de Arquitectura (Bogotá): arquitectura, diseño, educación arquitectónica, proyecto y construcción, urbanismo.

Idiomas de publicación: español, inglés, portugués y francés. Título abreviado: Rev. Arquit.

Titulo corto: RevArq

\section{Políticas de sección}

La revista se estructura en tres secciones correspondientes a las líneas de investigación activas y aprobadas por la institución, y dos complementarias, que presentan dinámicas propias de la Facultad de Diseño y las publicaciones relacionadas con la disciplina.

Cultura y espacio urbano. En esta sección se publican los artículos que se refieren a fenómenos sociales en relación con el espacio urbano, atendiendo aspectos de la historia, el patrimonio cultural y físico, y la estructura formal de las ciudades y el territorio.

Proyecto arquitectónico y urbano. En esta sección se presentan artículos sobre el concepto de proyecto, entendido como elemento que define y orienta las condiciones proyectuales que devienen en los hechos arquitectónicos o urbanos, y la forma como estos se convierten en un proceso de investigación y nuevo de conocimiento. También se presentan proyectos que sean resultados de investigación, los cuales se validan por medio de la ejecución y transformación en obra construida del proceso investigativo. También se contempla la publicación de investigaciones relacionadas con la pedagogía y didáctica de la arquitectura, el urbanismo y el diseño.

Tecnología, medioambiente y sostenibilidad. En esta sección se presentan artículos acerca de sistemas estructurales, materiales y procesos constructivos, medioambiente y gestión, relacionados con los entornos social-cultural, ecológico y económico.

Desde la Facultad. En esta sección se publican artículos generados en la Facultad de Diseño, relacionados con las actividades de docencia, extensión, formación en investigación o internacionalización, las cuales son reflejo de la dinámica y de las actividades realizadas por docentes, estudiantes y egresados; esta sección no puede superar el $20 \%$ del contenido.

Textos. En esta sección se publican reseñas, traducciones y memorias de eventos relacionados con las publicaciones en Arquitectura y Urbanismo.
A Frecuencia de publicación

Desde 1999 y hasta el 2015, la Revista de Arquitectura (Bogotá) publicó un volumen al año, a partir del 2016 se publicarán dos números por año en periodo anticipado, enero-junio y julio-diciembre, pero también maneja la publicación anticipada en línea de los artículos aceptados (versión Post-print del autor).

La Revista de Arquitectura (Bogotá) se divulga mediante versiones digitales (PDF, HTML, EPUB, XML) e impresascon un tiraje de 700 ejemplares, los tiempos de producción de estas versiones dependerán de los cronogramas establecidos por la editorial.

Los tiempos de recepción-revisión-aceptación pueden tardar entre seis y doce meses dependiendo del flujo editorial de cada sección y del proceso de revisión y edición adelantado.

Con el usuario y contraseña asignados, los autores pueden ingresar a la plataforma de gestión editorial y verificar el estado de revisión, edición o publicación del artículo.
A Canje

La Revista de Arquitectura (Bogotá) está interesada en establecer canje con publicaciones académicas, profesionales o científicas del área de Arquitectura y Urbanismo, como medio de reconocimiento y discusión de la producción científica en el campo de acción de la publicación.

Mecanismo

Para establecer canje por favor descargar, diligenciar y enviar el formato: RevArq FP20 Canjes

Universidad Católica de Colombia (2021,
junio-diciembre). Revista de
Arquitectura (Bogotá), 23(2),
I-132. Doi: 10.14718
ISSN: I657-0308
E-ISSN: 2357-626X
Especificaciones:
Formato: $34 \times 24 \mathrm{~cm}$
Papel: Mate II5 g
Tintas: Negro y policromía

A Contacto

Dirección postal:

Avenida Caracas N ${ }^{0} \cdot 46-72$ Universidad Católica de Colombia Bogotá D. C., Colombia Código postal: 111311

Facultad de Diseño Centro de Investigaciones (CIFAR) Sede El Claustro. Bloque "L", 4 piso Diag. 46A N ${ }^{0 .} 15 b-10$ Editor, Arq. César Eligio-Triana

Teléfonos:

+57 (1) $3277300-3277333$

Ext. $3109 ; 3112$ o 5146
Fax: +57 (1) 2858895

Correo electrónico:

revistadearquitectura@ucatolica.edu.co cifar@ucatolica.edu.co

Página WEB:

www.ucatolica.edu.co

Vínculo Revistas científicas

http://publicaciones.ucatolica.edu.co revistas-cientificas

https://revistadearquitectura.ucatolica.edu.co/ 
